\title{
On the onset of turbulence in natural convection on inclined plates
}

\author{
A. Rodríguez-Sevillano ${ }^{a}$, I. Pérez-Grande ${ }^{\mathrm{b}, *}$, J. Meseguer $^{\mathrm{b}}$ \\ ${ }^{a}$ Escuela Universitaria de Ingeniería Técnica Aeronáutica, Universidad Politécnica de Madrid, 28040 Madrid, Spain \\ b IDR/UPM, Escuela Técnica Superior de Ingenieros Aeronâuticos, Universidad Politécnica de Madrid, 28040 Madrid, Spain
}

\begin{abstract}
The problem of determination of the turbulence onset in natural convection on heated inclined plates in an air environment has been experimentally revisited. The transition has been detected by using hot wire velocity measurements. The onset of turbulence has been considered to take place where velocity fluctuations (measured through turbulence intensity) start to grow. Experiments have shown that the onset depends not only on the Grashof number defined in terms of the temperature difference between the heated plate and the surrounding air. A correlation between dimensionless Grashof and Reynolds numbers has been obtained, fitting quite well the experimental data.
\end{abstract}

\section{Introduction}

Natural convection on heated inclined plates has been widely studied, both analytically and experimentally, aiming mainly to determine the Nusselt number and, in turn, the heat transfer, for it being of great interest in many applications ranging for example from small electronic components to huge solar panels. Other applications are not only concerned with the magnitude of the heat transfer but with the flow regime, as explained below. In fact, one of the tasks in the design process of optical telescopes is to determine the thermal control needed to maintain the images of mirrors and their relative positions within the required tolerances, regardless of variations in the environmental conditions. The design of almost every telescope element requires a thermal analysis; small temperature differences in the optical devices or mounting structures might affect the alignment and mirror figures [1].

Apart from the thermal behavior of the telescope parts themselves, the quality of images is also affected by ambient temperature. The turbulence encountered by the light rays in the local telescope environment degrades its optical performance. This phenomenon is called 'seeing', and consists of optical aberrations produced by density non-homogeneities in the air along the optical path. The refraction index of air changes due to thermal gradients so that the wavefront incident on the mirror is randomly distorted, and therefore, images are altered. In particular, the seeing effect in the telescope mirror is called 'mirror seeing'.

Mirror seeing is therefore the result of natural convection on the mirror surface, for it being warmer than the surrounding air.
The effect is generated in the region where the temperature fluctuations are the largest and most intermittent, that is, just above the viscous conductive layer. The seeing effect has been qualitative and quantitatively studied [2,3], and a number of experiments have been carried out to measure it [4].

As it is well known [5], when a vertical or inclined plate is warmer than the environment, an ascending thermal boundary layer is formed. Its initial development is laminar, but at some distance from the leading edge turbulent eddies are formed and the transition to a turbulent boundary layer begins. Further up the flow become fully turbulent. Most of the image degradation in telescopes occurs in a thin but very turbulent layer above the surface. So, in order to minimise the mirror seeing effect, the air flow over the mirror has to be laminar, the optical effects being this way small and, what is more important, predictable. Therefore, part of the thermal feasibility study of a telescope should consist of determining whether the convective flow is going to be laminar or turbulent, in this last case being necessary to cool down the mirror to diminish the difference of temperature between mirror and air to keep the laminar regime. It has to be noticed that this mirror seeing effect is much more important in solar telescopes, for the primary mirror being directly facing to and absorbing energy from Sun, what could heat the surface noticeably. Although the study of the transition from laminar to turbulent regime in natural convection over inclined surfaces has many applications and is of great importance in many other fields, this work has been carried out in the context of the thermal study of a solar telescope (like the balloon-borne telescope Sunrise, flown from Esrange, the Swedish Space Corporation Balloon Facility, in June 8th, 2009 [6]). It was of great interest to determine when the transition from laminar to turbulent in a heated inclined surface occurred, aiming with this 
information to improve the design, either by avoiding the turbulent regime or at least by predicting the effects and the area it could affect.

The mechanism of natural convection is well understood, however, the complexities of fluid motion make it very difficult to obtain analytical solutions, unless the equations are solved under some simplifying assumptions with the consequent lack of generality. Over the last decades, the transition for inclined plates has been widely studied by several authors. An extensive and well focussed work was carried out by Tritton [7]. Unfortunately, the experiments were limited and the reliability of his results was questioned, among other reasons for some of the observations not being repeatable. Vliet [8] and Vliet and Ross [9] studied the transition for different inclinations with the assumption of constant heat flow. In this case most of the measurements were done on water and only the case of an inclination of $45^{\circ}$ was carried out with air. In these experiments, the transition was inferred from the characteristics of the plate temperature variation. Laminar flow was assumed to terminate where the plate temperature levelled out or reached a weak maximum as it did in some cases. This criterion for the determination of the onset of transition based on temperature measurements was then already questioned. So, for vertical natural convection flows, Jaluria and Gebhart [10] found that taking the wall temperature distribution for determining the boundaries of the transition regime excludes much of the actual transitional phenomenon in the boundary region since the wall temperature distribution shows a drop much further downstream of the appearance of several events that characterize transition, like turbulent bursts and thickening of the boundary region.

Lloyd and Sparrow [11] used electrochemical flow visualization techniques to determine the transition. Their experiments were carried out with water. Shaukatullah and Gebhardt [12] proved that the longitudinal vortices have a significant influence on the local heat transfer distribution during the laminar-turbulent transition. Later on, these vortices were experimentally studied by Zuercher et al. [13], using schlieren and PIV techniques.

More recently, Biertümpfel and Beer [14] studied the heat transfer increase in the laminar to turbulent transition in the presence of unsteady vortices. In this case the work was focussed on the determination of heat transfer rather than studying the onset of the transition itself. Their experiments were carried out just for a plate inclination of $26^{\circ}$ with respect to the vertical.

After reviewing the literature, with several tens of works published about this subject, one realizes that with the available data it is not a trivial issue to find out whether for a determined heated plate with a given inclination, turbulence is present or not. It has been widely accepted that the onset of the transition can be characterized by just the critical Grashof number, which depends for inclined plates on the angle of inclination of the plate. For instance, in a number of extensively used heat transfer textbooks as Holman [15], the values reported for the critical Grashof numbers are $5 \times 10^{9}, 2 \times 10^{9}, 10^{8}$ and $10^{6}$ for inclinations of $15^{\circ}, 30^{\circ}, 60^{\circ}$ and $70^{\circ}$ measured from the vertical, respectively. These values were obtained from Vliet and Ross [9] through temperature measurements. Reviewing the published works, it can be seen that the scattering of results about the onset of the transition is so wide that it only gives rough orders of magnitude of the values of critical Grashof numbers. Note that when using these data, a change of an order of magnitude in the critical Grashof number, leads to a factor of two in the length of plate available with laminar regime. Furthermore, although the Grashof number is undoubtedly the most relevant parameter to identify the onset, for vertical plates Jaluria and Gebhart [10] and more recently Jannot and Kunc [16] already identified the dependence of the onset of transition on other parameters, in that case on the critical height. The latter presented a remarkable and updated review for the vertical plates.
Regarding the analytical and numerical approach to the problem, a number of works can also be found in the literature. Since the scope of this work is merely experimental the review of the literature is not presented here. As example among the recent published works, Tumin [17] deals with the spatial stability of the flow.

Taking into account all the considerations described above, what has been aimed in this work is to determine the onset of transition from laminar to turbulent flow for heated inclined plates in an air environment. To do it, aiming to avoid the problems described in the previous paragraphs regarding the use of temperature measurements to determine the onset location, and given that schlieren techniques cannot be used for any fluid, velocities have been measured in this work. The amplification of the rms-values of velocity fluctuations has been taken as an indication of the appearance of turbulence. Indeed, the results are presented with the awareness that this location of the transition cannot be accurately determined, because the growth of the perturbations is gradual. Nevertheless, the change of behavior of the velocity fluctuations allows identifying where transition occurs. Temperatures have also been measured, but in order to characterize the flow and fluid properties.

In the following sections, the experimental setup, the experimental procedure and the results obtained are presented. As already stated by Tritton [7] and later on by Jaluria and Gebhart [10] transition has been found to be correlated not only with the Grashof number but also with the distance of the leading edge, $L$, where turbulence appears. Very satisfactory correlations have been obtained when the Reynolds number is taken into account as one of the variables for the correlations.

\section{Experimental apparatus}

The experimental apparatus, as sketched in Fig. 1, consists of a thermal blanket or more specifically a silicone rubber heater (1) supported by a substratum made of plywood (2), which is hinged to a table (3) in such a way that its tilting angle $\alpha$ can be fixed at any desired value between $0^{\circ}$ and $90^{\circ}$. A translation stage (4) is fixed to the substratum. The translation stage moves an arm (5) which in turn supports the hot-wire probe (6) used to measure air velocity close to the upper surface of the heated surface. The translation stage is placed perpendicular to the bottom side of the blanket, so the probe is displaced parallel to the $x$ axis (Fig. 1). The lateral position of the probe as well as its vertical distance to the blanket surface can be selected by changing the length of the arm (5), although in the experiments here reported both distances have been kept constant (the probe moves over the mid line of the blanket, at $25 \mathrm{~cm}$ from the lateral sides, and at a distance of

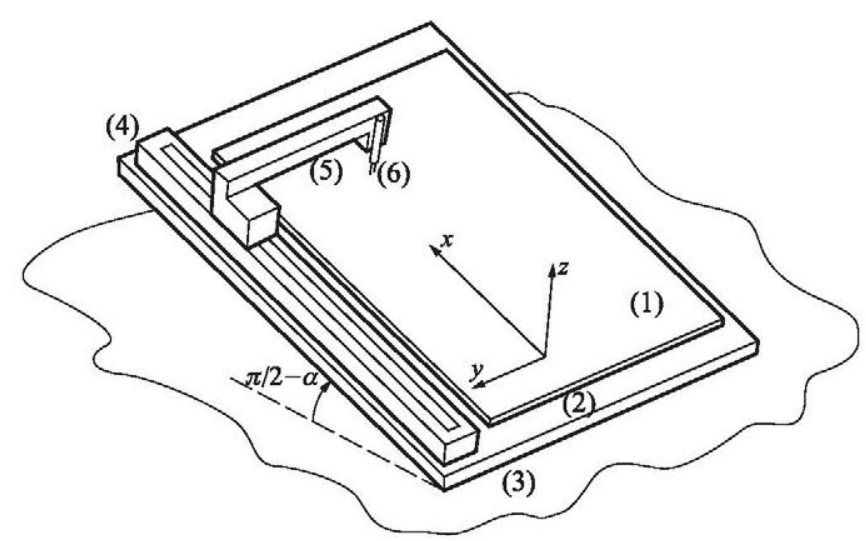

Fig. 1. Sketch of the experimental apparatus: thermal blanket (1), substratum (2), table (3), linear displacement guide (4), arm (5), and hot-wire probe (6). 
$2 \mathrm{~mm}$ over the blanket surface). To avoid undesired perturbations that could force the onset of turbulence on the thermal boundary layer of the plate, the whole described apparatus is placed inside an almost tight chamber whose base is a rectangle $1090 \mathrm{~mm}$ long, $880 \mathrm{~mm}$ wide and $1190 \mathrm{~mm}$ high. There are five holes $10 \mathrm{~mm}$ in diameter at the ceiling of the test chamber to allow the air inside the test chamber to reach pressure equilibrium with the ambient.

The thermal blanket is made of glass-fibre reinforced silicone rubber with an electrical resistance foil embedded. The thermal blanket is squared, $L_{b}=500 \mathrm{~mm}$ side and $0.5 \mathrm{~mm}$ thick. The blanket temperature is controlled through a Watlow PID SD (1/16 DIN) series controller, which allows the blanket temperature $T_{b}$ to be kept between $277 \mathrm{~K}$ and $423 \mathrm{~K}$ with $\pm 0.2 \mathrm{~K}$ temperature stability. Air temperature, $T_{a}$, is measured at $10 \mathrm{~mm}$ over the thermal blanket by using a thermocouple placed close to the top edge of the blanket, at the mid line, and at the wall of the tight chamber, $T_{w}$, some $800 \mathrm{~mm}$ over the tight chamber floor, with a thermometer (this chamber temperature is only used to establish when a steady state is reached in each experimental sequence).

The translation stage is a TSR-450B model from Zaber, the maximum displacement is $450 \mathrm{~mm}$, the position of the measuring probe being fixed with $\pm 68 \mu \mathrm{m}$ accuracy. For velocity measurements a Dantec Dynamics 55P0161 hot wire anemometer has been used. The hot-wire probe being a Dantec 55P16, equipped with a $5 \mu \mathrm{m}$ in diameter Platinum-Tungsten wire. The wire probe is parallel to the $y$ axis, so velocities normal to the wire (those contained in the $x z$ plane) are measured.

\section{Experimental procedure}

Experiments were performed as follows: once the desired angle of inclination of the tilting platform $\alpha$ is set and the hot-wire probe is positioned at the lower edge of the blanket, electric power is supplied to the thermal blanket so that the heating of the blanket starts. The temperature of the blanket is set to a value $T_{b}$. As already stated, temperature both close to the blanket surface and at the test chamber wall ( $T_{a}$ and $T_{w}$, respectively) were continuously measured to determine when a steady state was reached (when recorded temperatures do not show change with time) at the desired blanket temperature. It must be pointed out that the time needed to reach such an steady state may be large depending on the difference $\Delta T_{b a}=T_{b}-T_{a}$ between the blanket temperature, $T_{b}$, and the air temperature, $T_{a}$; such a time can be up to $2 \mathrm{~h}$ for large values of the temperature differences $\Delta T_{b a}$ (that means large values of the blanket temperature). The effect of thermal radiation on temperature measurements was estimated, resulting values lower than half degree in most of the cases.

Velocity measurements start once the steady state is reached. Then the hot-wire probe moves along the $x$ axis at $10 \mathrm{~mm}$ steps, and at each measuring position, after waiting for a few seconds to avoid perturbations (if any) due to the probe displacement, velocity is sampled at $10 \mathrm{kHz}$ during $1 \mathrm{~s}$. As already stated the hot wire is placed parallel to the $y$ axis, so that the velocity component in the plane normal to the wire direction is obtained (the plane $x z$ ). Obviously the phenomenon under study depends not only on the distance from the bottom blanket edge to the measuring point, but also on the distance $h$ from the probe to the blanket surface; in the following only the results corresponding to $h=2 \mathrm{~mm}$ are analyzed. This value has been selected as the most suitable to characterize the phenomenon here studied, after measuring velocities at different distances. New measurement campaigns at intermediate distances are envisaged in order to get a better description of the boundary layer development over the blanket.

In order to check the repeatability of the experiment, the above described procedure is carried out at least five times for each pair of values of both the inclination angle $\alpha$ (measured from the vertical) and the blanket temperature $T_{b}$. In general, the different runs within a five-run set were not performed in a unique experimental session, by performing two consecutive runs just one after another, but allowing a gap of several hours between two consecutive runs.

\section{Results}

As explained in the previous section, records of velocities have been obtained for different inclination angles, $\alpha$, and blanket temperatures, $T_{b}$ (note that, as defined in Fig. $1, \alpha=0^{\circ}$ means that the plate is vertical). From these data the mean velocity at each position, $U(x)$, as well as the standard deviation or root mean square value, $\sigma(x)$, are calculated, the turbulence intensity being $I_{u}(x)=$ $\sigma(x) / U(x)$.

The results of a typical five-run campaign are shown in Fig. 2. These particular results, presented to illustrate the measurements, correspond to an inclination $\alpha=30^{\circ}$ and a blanket temperature $T_{b}=70^{\circ} \mathrm{C}$. In Fig. 2 the averaged values obtained from the five different runs performed for the selected values of $\alpha$ and $T_{b}$ are shown. Note that the scales for the velocity $U$ and for the turbulence intensity $I_{u}$ are rather different; in both cases an indication of the scattering of the experimental results is also given. Concerning mean velocity, measured results are to a great extent repetitive, the mean velocity curves being practically equal no matter the run under consideration is. This happens when the angle $\alpha$ of the heated surface is small but it is no longer valid for large values of $\alpha$ (when the plate is close to the horizontal), where the mean velocity curves change from one run to another (although basically the shape of the velocity curves is the same for the different runs within the same five-run set). Therefore, at high values of the inclination angle $\alpha$ different averaged velocities have been obtained within the same five-run set, nevertheless, such a tendency does not hold in the case of the standard deviation $\sigma$, being the values quite repeatable. It must be stressed that the sharp leading edge of the thermal blanket was not provided with any faring to smooth it, which could slightly affect the normal development of the boundary layer close to the leading edge, mainly at high values of the inclination angle.

In the same Fig. 2 the results corresponding to the turbulence intensity are depicted. In spite of the scattering of the experimental data, the results represented in Fig. 2 show that close to the bottom edge of the blanket the measured turbulence intensity $I_{u}$ is within a narrow band, the mean value of the turbulence intensity in this region being small and constant or almost constant; once surpassed some threshold value of the distance to the bottom edge the turbu-

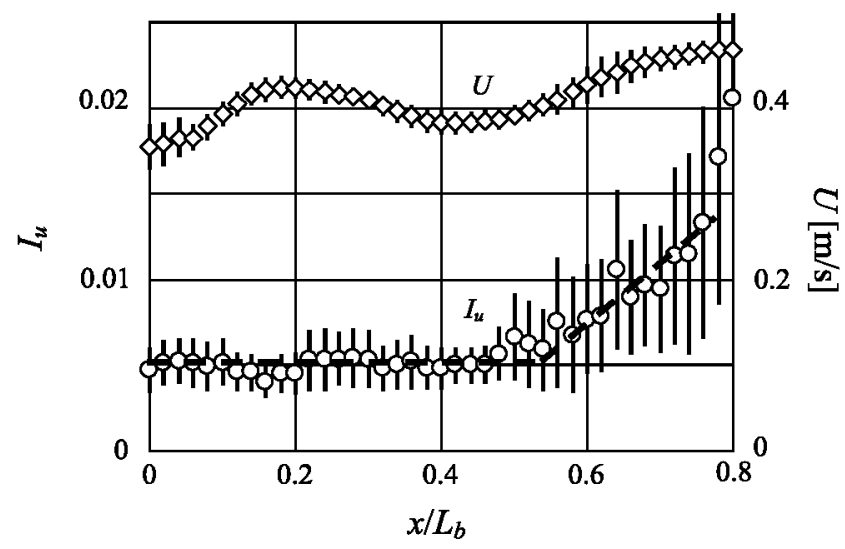

Fig. 2. Variation with the dimensionless distance from the bottom edge of the blanket, $x / L_{b}$, of the mean velocity, $U$, and the turbulence intensity, $I_{u}$. The results correspond to an inclination angle $\alpha=30^{\circ}$ and a blanket temperature $T_{b}=70^{\circ} \mathrm{C}$. 
lence intensity $I_{u}$ starts to grow almost linearly at the beginning and much more pronouncedly at larger distances. To enhance the perception of the behavior of the turbulence intensity, these regions of constant value of $I_{u}$ and of linear growth have been identified with dashed lines in Fig. 2. The criterion adopted to define the distance $L$ where the onset of turbulence takes place is just the point where the turbulence intensity starts to grow linearly, which in the case shown if Fig. 2 corresponds to $L / L_{b} \cong 0.5$, although due to data scattering such point can be only roughly defined (say $L / L_{b}=0.5 \pm 0.05$ ).

From the different records like the ones presented in Fig. 2, corresponding to the different experimental configurations indentified by the parameters $\alpha, T_{b}$, the distance from the leading edge $L\left(\alpha, T_{b}\right)$ where turbulence starts is determined. Indeed, as already mentioned, the determination of the onset of turbulence is not a well defined task, because fluctuations start to grow gradually. Nevertheless, in most of the experimental results there is a region close to the blanket leading edge where turbulence intensity is very small and constant or almost constant, and there is another region where turbulence intensity grows almost linearly. Therefore, the distance from the plate leading edge where these two regions match is defined as the distance $L$ where the onset of turbulence is considered to take place.

Since the driven mechanism of the fluid motion is the difference between blanket and air temperatures, $\Delta T_{b a}=T_{b}-T_{a}$, the variation of the dimensionless distance $L / L_{b}$ with the angle of inclination $\alpha$ and the temperature jump $\Delta T_{b a}$ is summarized in Fig. 3. In this plot the length is presented in dimensionless form, the length of the blanket $L_{b}$ being used for this purpose. Obviously, the larger the temperature difference $\Delta T_{b a}$ and the larger the angle $\alpha$ the smaller is the distance $L / L_{b}$ where turbulence starts.

Once determined the distance $L$ where turbulence starts, the Grashof number at this distance $\mathrm{Gr}=g \beta \Delta T_{b a} L^{3} / v^{2}$ has been calculated; in this expression $g$ stands for the gravity acceleration $\left(g=9.81 \mathrm{~m} / \mathrm{s}^{2}\right), \beta$ for the air thermal expansion coefficient, whose variation with temperature, within the interval of temperatures under consideration, is $\beta=\left(3.583-0.0089 T_{m}\right) 10^{-3} \mathrm{~K}^{-1}$, and $v$ is the kinematic viscosity of air, whose temperature variation within the considered interval of temperatures is $v=(1.294+$ $\left.0.01 T_{m}\right) 10^{-5} \mathrm{~m}^{2} / \mathrm{s}$; in these expressions for $\beta$ and $v T_{m}$ is a mean temperature defined as $T_{m}=\left(T_{b}+T_{a}\right) / 2$. The variation of the dimensionless distance with the Grashof number is shown in Fig. 4. From this plot it seems that the values of $L / L_{b}$ corresponding to the different blanket inclinations collapse in one single curve when the Grashof number is used instead of the temperature difference

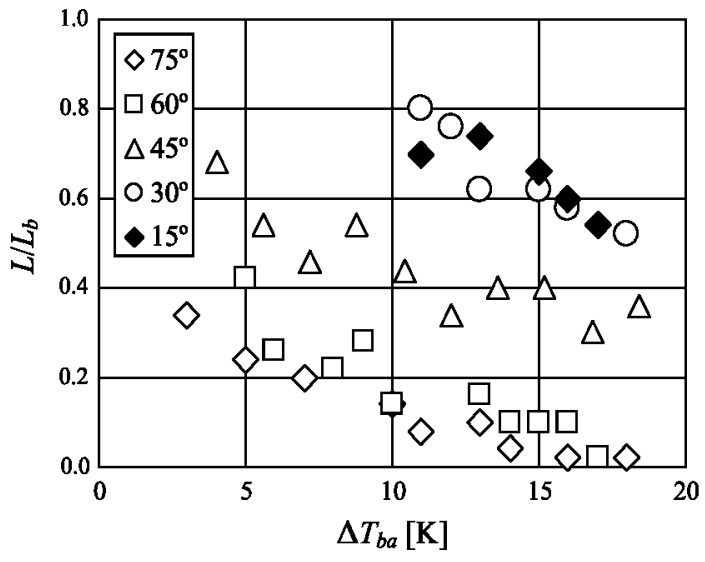

Fig. 3. Variation with the temperature difference between the blanket temperature and the air temperature, $\triangle T_{b a}=T_{b}-T_{a}$, of the dimensionless distance from the bottom edge of the blanket where the onset of turbulence takes place, $L / L_{b}$, symbols identify the inclination angle $\alpha$ of the thermal blanket as indicated in the inset.

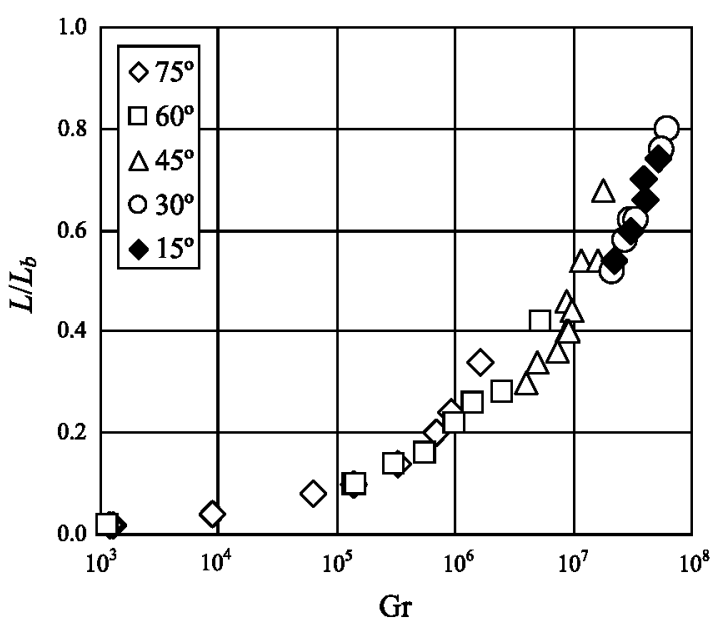

Fig. 4. Variation with the Grashof number, Gr, of the dimensionless distance from the bottom edge of the blanket where the onset of turbulence takes place, $L L_{b}$, symbols identify the inclination angle $\alpha$ of the thermal blanket as indicated in the inset.

$\Delta T_{b a}$. It can be noticed that, as already explained in Section 1, the Grashof numbers here obtained for the transition are somehow lower than the reported when the values are calculated from temperatures measurements.

According to Jaluria and Gebhart [10], who analyzed the transition from laminar to turbulent flow of natural convection of water close to a vertical plate uniformly heated, transition events are not correlated in terms of the Grashof number, but in terms of the ratio between this parameter and a power of the distance from the plate edge to the transition point, say $\mathrm{Gr} / L^{m}$, where $m$ is of order $1 / 2$. Assuming here a similar behavior, in order to find a better correlation, the Reynolds number $\operatorname{Re}=U L / v$ has been calculated (where $U$ stands for the average flow velocity at a distance $L$ from the edge of the blanket), and the parameter $\operatorname{Re}\left(L / L_{b}\right)^{n}$ has been considered instead of $L$. Note that the use of $L / L_{b}$ instead of $L$, since $L_{b}$ (the length of the thermal blanket) is not a real characteristic length of the problem, is only a way to make dimensionless the experimental results. The variation of the Reynolds number multiplied by $\left(L / L_{b}\right)^{n}$ with the Grashof number is depicted in Fig. 5. As can be seen in the figure, a very satisfactory correlation between the parameters

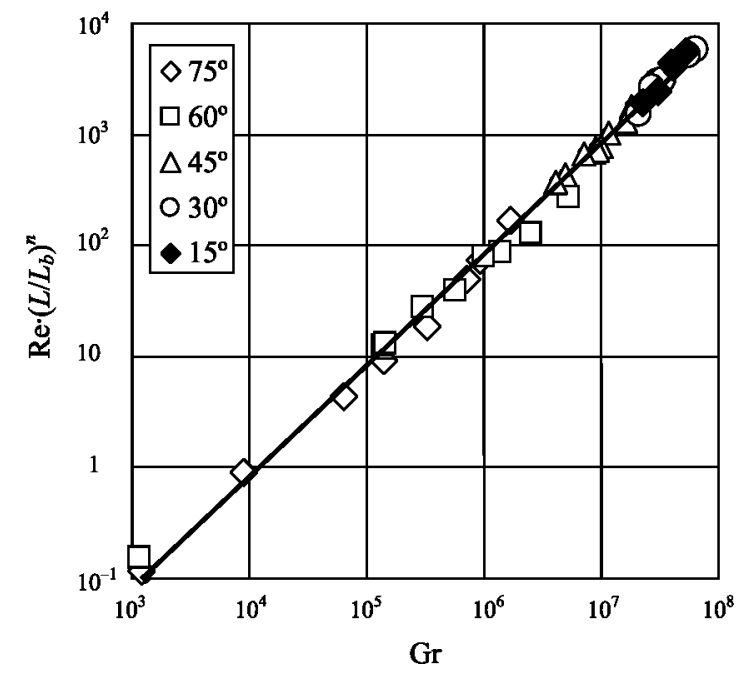

Fig. 5. Variation with the Grashof number, Gr, of the parameter $\operatorname{Re}\left(L / L_{b}\right)^{n}$, with $n=1.761$. Symbols identify the inclination angle $\alpha$ of the thermal blanket as indicated in the inset. 
has been obtained, the dependence of Grashof number on the parameter $\operatorname{Re}\left(L / L_{b}\right)^{n}$ being almost linear when $n \cong 3^{1 / 2}$; the best fitting of the linear approximation to the experimental being for $n=1.761$, when the regression coefficient becomes $R^{2}=0.996$.

Although the results shown in Fig. 5 are restricted to a separation $h=2 \mathrm{~mm}$ from the blanket surface, they include all the main features of the phenomenon under consideration: there is a region close to the heated surface edge where the flow is laminar, so that seeing effects are negligible in this region. After some distance from the leading edge (whose magnitude depends on the inclination of the plate), flow instabilities start to grow and the flow regime in the boundary layer becomes turbulent, thus causing a noticeable increment of seeing effect. According to Fig. 5 the dependence of $\operatorname{Re}\left(L / L_{b}\right)^{n}$ on the Grashof number is almost linear, $\operatorname{Re}\left(L / L_{b}\right)^{n} \cong k \mathrm{Gr}$, with $k \cong 10^{-4}$.

\section{Conclusions}

The onset of the turbulence on a thermal boundary layer on a flat inclined plate has been experimentally analyzed by hot wire measuring of the velocities over the plate. The distance from the leading edge where turbulence intensity starts to grow (which has been adopted as an indication of the transition from laminar to turbulence regime occurs) has been determined for different temperatures of the plate and for different inclination angles. Although results are restricted to a given height of the measuring probe (the hot wire was $2 \mathrm{~mm}$ over the plate), experimental results behave as expected: the measured length increases as both the plate inclination angle (measured with respect to the vertical position) and the plate temperature decrease.

From experimental results dimensionless parameters, Grashof number and Reynolds number, have been calculated, and a remarkable correlation has been obtained when the Grashof number is considered as a function of the product of the Reynolds number by a given power of the dimensionless distance $\left(L / L_{b}\right)^{n}$.

\section{Acknowledgements}

This work has been supported by the Universidad Politécnica de Madrid, under the program "Primera Convocatoria de Apoyo al
Doctorado en las Escuelas Universitarias de la UPM" and by the Spanish Ministerio de Educación y Ciencia, Project ESP200613030-C06-05.

\section{References}

P.Y. Bely, The Design and Construction of Large Optical Telescopes, Springer Verlag, Berlin, 2002

I.M. Beckers, J. Melnick, Effects of heat sources in the telescope beam on astronomical image quality, SPIE Proc. 2199 (1994) 478-480.

L. Zago, An engineering formulae for local and dome seeing, SPIE Proc. 2871 (1996) 726-736

M.T. Bridgeland, C.R. Jenkins, Measurement of mirror seeing in the laboratory and at the telescope, New Astron. Rev. 42 (1998) 435-440.

E.R.G. Eckert, R.M. Drake, Analysis of Heat and Mass Transfer, Hemisphere Publishing Corp., New York, 1987.

P. Barthol, A.M. Gandorfer, S.K. Solanki, M. Knölker, V. Martinez Pillet, W. Schmidt, A.M. Title, The SUNRISE Team, SUNRISE: high resolution UV/VIS observations of the Sun from the stratosphere, Adv. Space Res. 42 (2008) 7077.

D.]. Tritton, Transition to turbulence in the free convection boundary layers on an inclined heated plate, J. Fluid Mech. 16 (1963) 417-435.

G.C. Vliet, Natural convection local heat transfer on constant-heat-flux inclined surfaces, J. Heat Trans.-Trans. ASME 91 (1969) 511-516.

G.C. Vliet, D.C. Ross, Turbulent natural convection on upward and downward facing inclined constant heat flux surfaces, J. Heat Trans.-Trans. ASME 97 (1975) 549-555.

Y. Jaluria, B. Gebhart, On transition mechanisms in vertical natural convection flow, J. Fluid Mech. 66 (1974) 309-337.

J.R. Lloyd, E.M. Sparrow, On instability of natural convection flow on inclined plates, J. Fluid Mech. 42 (1970) 465-470.

H. Shaukatullah, B. Gebhart, An experimental investigation of natura convection flow on an inclined surface, Int. J. Heat Mass Trans. 21 (1978) 1481-1490.

E.J. Zuercher, J.W. Jacobs, C.F. Chen, Experimental study of the stability of boundary-layer flow along a heated, inclined plate, J. Fluid Mech. 367 (1998) $1-25$.

R. Biertümpfel, H. Beer, Natural convection heat transfer increase at the laminar-turbulent transition in the presence of instationary longitudinal vortices, Int. J. Heat Mass Trans. 46 (2003) 3109-3117.

J.P. Holman, Heat Transfer, eighth ed., McGraw-Hill, New York, 1997.

M. Jannot, T. Kunc, Onset of transition to turbulence in natural convection with gas along a vertical isotherm plane, Int. J. Heat Mass Trans. 41 (1998) $4327-$ 4340 .

A. Tumin, The spatial stability of natural convection flow on inclined plates, J. Fluid Eng.-Trans. ASME 125 (2003) 428-437. 